\title{
Recurrent Abdominal Pain Diagnosed as Kimura's Disease
}

\author{
Airu Liu, Jing Li, Duanmin Hu, Ruidong Chen, Wen Tang \\ Department of Gastroenterology, The Second Hospital of Soochow University, Soochow, Jiangsu China
}
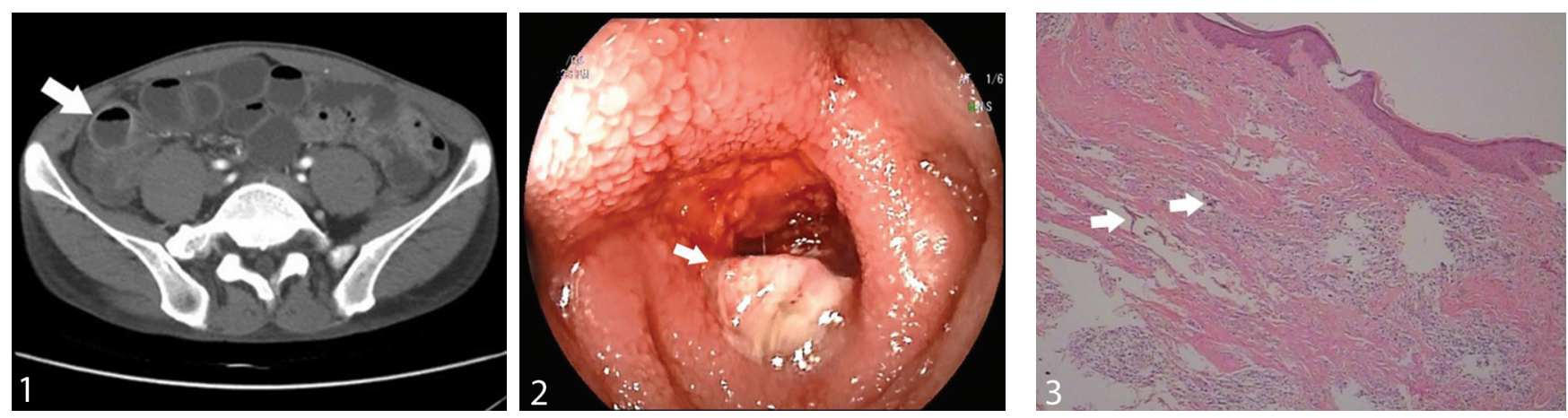

A 50-year old Chinese man was admitted for recurrent periumbilical abdominal pain present in the last year. He presented multiple subcutaneous asymptomatic masses located on head and neck, the largest being $3 \mathrm{~cm}$ in diameter. The laboratory tests showed elevated peripheral blood eosinophilia, immunoglobulin (lg) E levels, significant albuminuria, with resultant hypoalbuminemia. Abdominal enhanced computed tomography revealed thick and hyperenhanced segmental small intestinal wall (Fig. 1). Double-balloon transanal enteroscopy passed into the upper ileum and revealed a semi-circumferential hyperplastic lesion with lumen stenosis and peripheral mucosal redness and swelling (Fig. 2). The histology depicted chronic inflammation in the submucosa, with a large eosinophilic infiltration. Brain Magnetic Resonance Imaging indicated multiple nodules of the subcutaneous soft tissue on head with mixed isohypointensity on T2-weighted images. A nodule biopsy detected marked lymphoid follicular hyperplasia with a large eosinophilic infiltration, fibrosis, and vascular proliferation (Fig. $3 \mathrm{H} \& \mathrm{E}, 100 \mathrm{x}$ ). A renal biopsy indicated membranous nephropathy stage II and serum antibody against phospholipase A2 receptor was negative. Finally, the patient was diagnosed with Kimura's disease (KD) affecting both the intestine and kidneys. After treatment with corticosteroids, the patient's condition significantly improved.

Kimura's disease is a rare, chronic inflammatory disorder that typically presents as painless subcutaneous tumor-like masses on the head and neck region. It is diagnosed based on the presence of a characteristic subcutaneous mass, elevated peripheral blood eosinophilia, Ig E levels and a biopsy that shows the abnormal proliferation of lymphoid follicles and vascular endothelium, with eosinophilic infiltration [1]. Indeed, multiple organs can be affected, with about $12-16 \%$ of patients suffering renal involvement, and nephrotic syndrome may be the main presentation [2]. Small bowel involvement has rarely been reported in the literature. Currently there is no consensus regarding the optimal treatment for KD. Treatment options include corticosteroids, surgical excision, radiotherapy and combined therapy. Corticosteroids are particularly applicable for cases of KD with renal involvement [3]. Although $\mathrm{KD}$ is benign, it is prone to recurrence.

\section{Corresponding author: Wen Tang, louisetangwen@163.com}

Conflicts of interest: None to declare.

\section{REFERENCES}

1. Armstrong WB, Allison G, Pena F, Kim JK. Kimura's disease: two case reports and a literature review. Ann Otol Rhinol Laryngol 1998;107:1066-1071. doi:10.1177/000348949810701212

2. Chen Y, Wang J, Xu F, Zeng C, Liu Z. Clinicopathological features and prognosis of Kimura's disease with renal involvement in Chinese patients. Clin Nephrol 2016;85:332-339. doi:10.5414/CN108749

3. Senel MF, Van Buren CT, Etheridge WB, Barcenas C, Jammal C, Kahan BD. Effects of cyclosporine, azathioprine and prednisone on Kimura's disease and focal segmental glomerulosclerosis in renal transplant patients. Clin Nephrol 1996;45:18-21. 